\title{
An unusual case report of a painless hard swelling in the angle of the mandible- An enigma in diagnosis
}

\author{
Beenaprecilla G. S. ${ }^{1, *}$, Prashanth Shenoy ${ }^{2}$, Laxmikanth Chatra ${ }^{3}$, Veena K. M. ${ }^{4}$, Rachana V. Prabhu ${ }^{5}$ \\ ${ }^{1}$ Post Graduate, ${ }^{2,4}$ Professor, ${ }^{3}$ Senior Professor \& HOD, ${ }^{5}$ Reader, Dept. of Oral Medicine \& Radiology, Yenepoya Dental \\ College, Yenepoya University, Mangalore, Karnataka, India
}

*Corresponding Author:

Email: beenashanmugam@gmail.com

\begin{abstract}
Asymptomatic bony hard swelling of the jaws includes a wide variety of disorders like developmental disorders, inflammatory, benign and malignant tumors of odontogenic/non odontogenic origin. A thorough history along with adequate clinical examination and investigations like biochemical, radiographic and histopathology most of the lesions can be assigned with reasonable certainty into one of several categories. The main aim of this present article is to discuss a case of a juvenile ossifying fibroma in a 11-year old male patient who presented with a bony hard swelling in the unusual site of left angle of the mandible which was symptom-free and present since 2 months.
\end{abstract}

Keywords: Ossifying fibroma, Juvenile ossifying fibroma, Radiographic features, Cone beam computed tomography.

\section{Introduction}

Ossifying fibroma is classified under the fibroosseous lesions of the jaws. It usually presents as a progressively growing lesion that can attain an enormous size with resultant deformity if left untreated The first description of ossifying fibroma was given by Menzel in 1872. ${ }^{1}$ In 1972, WHO classified ossifying fibroma into two types as ossifying fibroma and cementifying fibromas based on the presence of bone or cementum; some authors regarded the cementifying fibroma as an odontogenic tumour and consider ossifying fibromas separately as non odontogenic neoplasms. ${ }^{2}$ This seems an arbitrary and unnecessary separation, as the clinical, radiologic, and prognostic features of the lesions are identical hence in 1992 WHO considered it in one heading as cemento ossifying fibroma. ${ }^{3}$ Further, the term "cemento ossifying fibroma" was replaced by "ossifying fibroma" in 2005 under the WHO classification as cementum-like material thought to be of dental origin was also found in fibroma occurring in extra-gnathic sites. ${ }^{3,4}$

DS MacDonald-Jankowski has done a systematic review of 781 cases and reported that OF affected females more frequently, and was three times more prevalent in the mandible premolar- molar region. The mean age at first presentation was 31 years. The decade with the greatest frequency was the fourth. ${ }^{5}$ In contrast the juvenile (aggressive) ossifying fibroma (JOF) mainly affects individuals younger than 15 years of age especially involving the paranasal sinuses, periorbital bones in $90 \%$ of cases and behaves in an aggressive fashion when compared to ossifying fibroma.

The present paper describes a rare case of juvenile ossifying fibroma at the angle of the mandible in a 11year-old male child with atypical clinical and radiographic presentation.

\section{Case Report}

A 11 year old male child reported with a chief complaint of swelling in the left angle of the mandible since 2 months. History revealed that swelling was first noticed about 2 months back, was sudden in onset, initially smaller and gradually increased to the present size. The swelling was not associated with any fever, pain, discharge, paresthesia, dryness of oral cavity or difficulty in mastication. The past medical, dental, and family history was non contributory. On general physical examination Right submandibular lymphnode was palpable, mobile, non tender and soft in consistency.

On extra oral examination, a well defined solitary swelling of size approximately $2.5 \mathrm{cms}$ was noted on the left angle of the mandible. Surface over the swelling and the surrounding areas appeared normal. No sinus opening/discharge noted (Fig. 1A). On palpation inspectory findings were confirmed regarding site, size and extent. It is non pulsatile, non tender, bony hard in consistency. The swelling was fixed to the underlying bone, overlying skin was movable. Intra orally there was no decayed teeth /swelling/vestibular obliteration noted (Fig. 1B). Salivary flow was also normal. Considering the slow growing nature of the lesion and its features, a clinical diagnosis of benign tumor of the jaw was given. As the erupting age of the third molar was not completed we cannot conclude whether it is of odontogenic/ non odontogenic origin. 


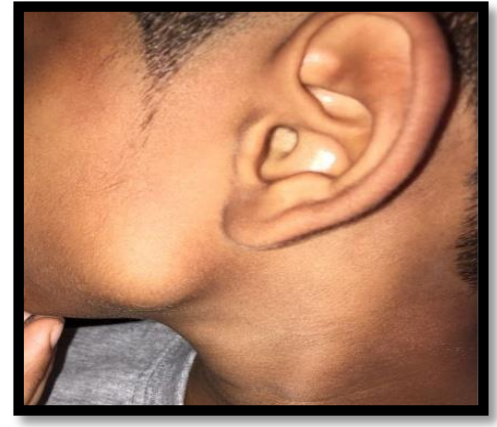

Fig. 1A

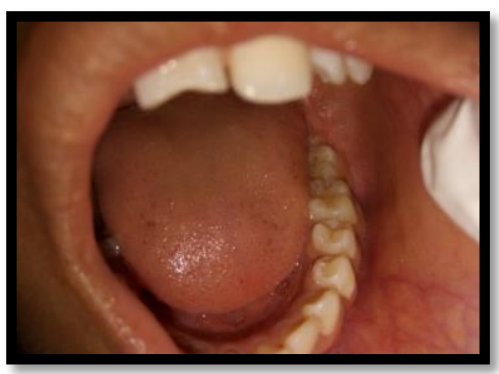

Fig. 1B

3D Imaging by CBCT was advised to identify the exact location, extent, internal structure and involvement of adjacent structures. It revealed a well defined osteolytic lesion on the left angle of the mandible measuring $2.5 \mathrm{cms}$ in size, Lesion was seen to be below the Inferior alveolar nerve canal. Crypt of 38 appeared to be intact. Lower border of the mandible was lost at the angle of the mandible. Periosteal bone reaction was noted. Discrete multiple flecks of radiopacities were also noted within the lesion (Fig. 2A). CBCT features suggested destructive pattern of the lesion with a sun ray appearance which raised the suspicion of malignancy. The most common malignant tumor in children affecting the jaws is Ewing's sarcoma, however the clinical symptoms like rapidly growing swelling, paresthesia, displacement/ loosening of teeth, weight loss etc were not present.

\section{CBCT Images:}

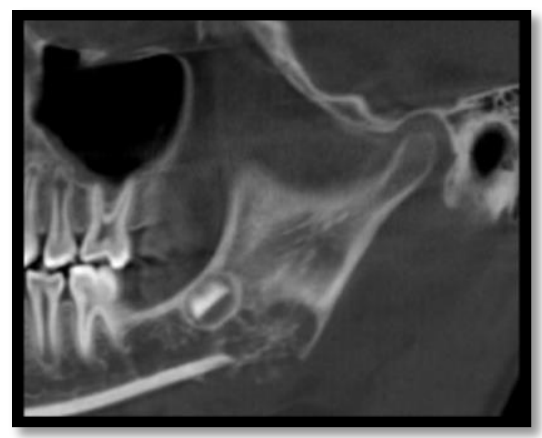

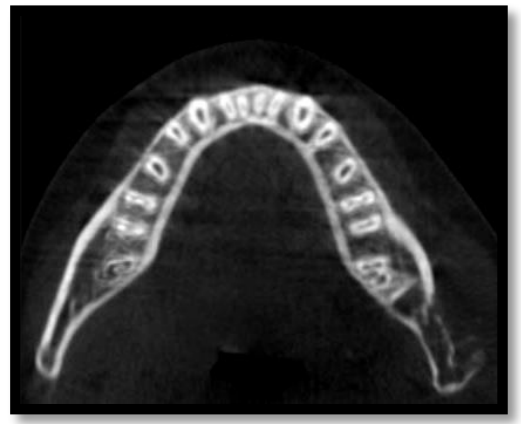

Fig. 2(B) - Axial view

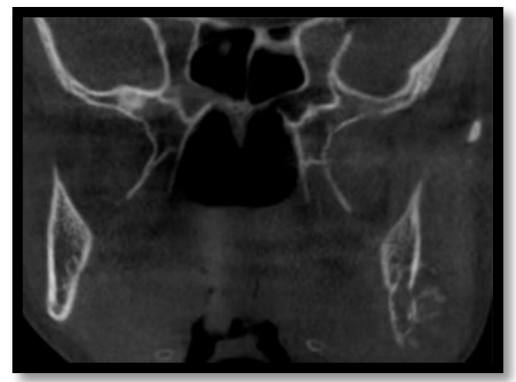

Fig. 2(C): Coronal view

Patient was referred to the department of oral and maxillofacial surgery where they consulted with the oncology team and incisional biopsy was done. Histopathological examination showed hypo and hypercellular fibroblstic stroma. The stromal cells showed hyperchromatic nuclei and moderate amount of cytoplasm. No marked atypia. Focal areas of peripheral tissue showed occasional osteoclast. Features were suggestive of ossifying fibroma. Resection of the lesion was done and sent for histopathological examination which revealed cellular fibrous stroma, tumor cells interspersed with band of osteoid having osteoblastic rimming along with poorly mineralized woven bone (Fig. 3). The impression of Juvenile aggressive ossifying fibroma was given. As juvenile ossifying fibroma shows high recurrence, patient is kept under observation for every six months for the next 2 years.

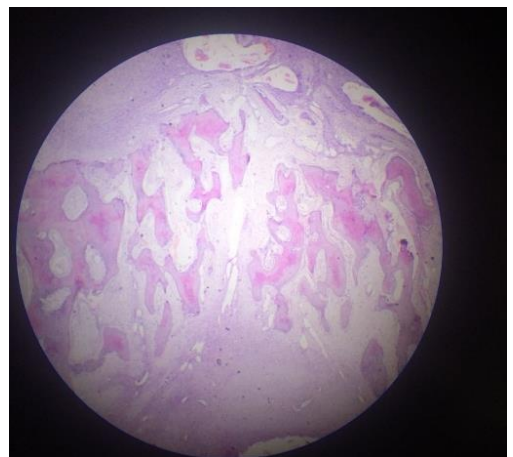

Fig.3: Micrograph of resected specimen

Fig. 2(A): Pan view 


\section{Discussion}

A lesion with a characteristics of a juvenile ossifying fibroma was first reported by Benjamin's in 1938. ${ }^{6}$ This lesion was located in the frontal sinus. The term "Juvenile Ossifying fibroma" was first used by Johnson in $1952^{7}$ to describe aggressive forms of ossifying fibroma that occurred in the craniofacial bones of children It is known by a variety of terms, , juvenile aggressive ossifying fibroma, trabecular osteo desmoblastoma, Juvenile active ossifying fibroma, and active fibrous dysplasia. ${ }^{8}$ It is a locally aggressive lesion and known to have recurrence rate from $30 \%$ to $58 \%$, and these are generally seen at early stage and these are more aggressive than the primary lesions however malignant transformation has not been reported in literature so far. ${ }^{9}$ This aggressive rate can be attributed to the increased rate of osteoblastic and osteoclastic activity in children but there are few cases of JOF reported in adults.

It is thought to develop from the multipotential mesenchymal cells of periodontal ligament origin. ${ }^{5}$ The aetiology is unknown but was thought to be odontogenic, developmental or traumatic origins and it is characterized by rapid growth .Maxilla is more commonly affected and Mandibular lesions are seen in only $10 \%$ of the cases. In the mandible, the angle and ramus are the most common sites of involvement. ${ }^{8}$ In our case there is no history of trauma or tooth involvement. It was slow growing and mandible was affected.

MacDonald-Jankowski ${ }^{5}$ described three stages in the radiographic appearance. $42 \%$ were radiolucent, $24 \%$ were radiopaque and $34 \%$ had mixed appearance. Three different patterns of radiographical borders were reported by $\mathrm{Su}$ et $a l^{8}{ }^{8} \mathrm{~A}$ defined lesion without a sclerotic border (40\%), a well-defined lesion with a sclerotic border (45\%), and a lesion with an ill-defined border $(15 \%)$.Our case showed a well defined lesion with mixed radiographic appearance and periosteal reaction which can be misdiagnosed and mismanaged because of its rapidly progressive and osteolytic nature. There is no consensus on the treatment of JOF cases. Radical resection, local excision conservatively or enucleation with curettage are among the treatment alternatives and long term follow up is mandatory.

\section{Conclusion}

Juvenile ossifying fibroma has different clinical and radiographic features can cause diagnostic dilemma. It should be considered as differential diagnosis when we encounter a painless slow growing bony hard swelling in the jaws with radiographic features as mentioned above. Careful evaluation of the clinical, radiological, and histological components of this lesion is needed to surmount the diagnostic and therapeutic challenges connected with it.

\section{References}

1. Swami AN, Kale LM, Mishra SS, Choudhary SH. Central ossifying fibroma of mandible: A case report and review of literature. J Ind Acad Oral Med Radiol 2015;27:131-5.

2. 2 Waldron C A. Fibro-osseous lesions of the jaws. J Oral Maxillofac Surg 1985;43:249-62.

3. Reichart PA, Philipsen HP, Sciubba JJ. The new classification of head and neck tumours [WHO]—any changes? Oral Oncol 2006;42:757-8.

4. Osunde, O., Iyogun, C., \& Adebola, R. (2013). Juvenile Aggressive Ossifying Fibroma of the Maxilla: A Case Report and Review of the Literature. Ann Med Health Sci Res 3(2),288-90. http://doi.org/10.4103/21419248.113685

5. Liu Y, Wang H, You M, Yang Z, Miao J, Shimizutani K, et al. Ossifying fi bromas of the jaw bone: 20 cases. Dentomaxillofac Radiol 2010;39:57-63.

6. DS MacDonald-Jankowski. Ossifying fibroma: a systematic review Dentomaxillofacial Radiol 2009;38:495-513.

7. Johnson LC et al Juvenile active ossifying fibroma: it's nature dynamics and origin. Acta Otolaryngol Suppl 1991;448:1

8. Bachani L, Lingappa A, Shivaprasad S. Juvenile trabecular ossifying fibroma involving mandibular angle and ramus region. Int J Med Dent Case Rep 2017;4:1-4.

9. Walter JM, Terry BC, Small EW, Matteson RR, Howell RM, Hill C (1979) Aggressive ossifying fibroma of the maxilla: a review of literature and report of a case. $\mathrm{J} \mathrm{Oral}$ Surg 37:76-286.

10. Eversole LR, Leider AS, Nelson K. Ossifying fibroma: A clinicopathologic study of sixty-four cases. Oral Surg Oral Med Oral Pathol 1985;60:505-11.

11. Su L, Weathers DR, Waldron CA. Distinguishing features of focal cemento-osseous dysplasia and cementoossifying fibromas. II. A clinical and radiologic spectrum of 316 cases. Oral Surg Oral Med Oral Pathol Oral Radiol Endod 1997;84:540-9. 